\title{
Gorlin-Goltz Syndrome: Literature Review and Report of Two Clinical Cases
}

\author{
Juliane Maria Iagnes Prestes ${ }^{1}$ and João Luiz Carlinii ${ }^{2 *}$ \\ ${ }^{1}$ Dentistry Student at the Federal University of Paraná, Paraná, Brazil \\ ${ }^{2}$ Professor of Oral and Maxillofacial Surgery and Traumatology I at the Federal \\ University of Paraná, Paraná, Brazil \\ *Corresponding Author: João Luiz Carlini, Professor of Oral and Maxillofacial \\ Surgery and Traumatology I at the Federal University of Paraná, Paraná, Brazil.
}

Received: April 01, 2021

Published: April 23, 2021

(C) All rights are reserved by Juliane Maria

Iagnes Prestes and João Luiz Carlini.

\begin{abstract}
The Gorlin-Goltz Syndrome, also known as Basal Cell Nevus Syndrome, is characterized by the triad of multiple basal cell carcinomas, keratocystic odontogenic tumors, and skeletal abnormalities. However, due to its variable expressiveness, other manifestations may occur, making diagnosis difficult. In this article, we aim to recall the signs and symptoms that can be identified in the bearer of this syndrome by reviewing the literature, alerting the health professional, and discussing their diagnostic process, illustrating it with two clinical cases from the Federal University of Paraná (UFPR). It was also intended to indicate its adequate treatment, since the management of this condition requires the monitoring of a multidisciplinary team in the long term, due to the involvement of multiple systems, the high rate of recurrence of the lesions, and the late appearance of carcinomas.
\end{abstract}

Keywords: Basal Cell Nevus Syndrome; Basal Cell Carcinoma; Gorlin-Goltz Syndrome; Odontogenic Cysts

\section{Introduction}

The Gorlin-Goltz Syndrome, also known as Basal Cell Nevus Syndrome (BCNS), is an autosomal dominant condition, with high penetrance and variable expressiveness. Although it is possible to identify reports in the literature of patients with symptoms suggestive of this syndrome since 1884, it was only in 1960 that Robert J. Gorlin and Robert W. Goltz recognized that the triad of basal cell carcinomas, keratocystic odontogenic tumors, and skeletal anomalies characterized a distinct entity $[1,2]$.

The prevalence of this syndrome varies according to the region studied and is estimated worldwide at about $1: 57,000$ to 1 : $256,000[1,3]$. It affects men and women in equal proportion, with the caucasian race, followed by the black race, being the most affected [4].

Currently, it is known that BCNS development results from mutations in the PTCH1, PTCH2, or SUFU genes, which are located, respectively, on chromosomes 9q22.32, 1p34.1, and 10q24.32.
The PTCH1 gene encodes a transmembrane glycoprotein, which acts as a tumor suppressor of the Hedhog (Hh) signaling pathway. This pathway is pro-oncogenic and uses several ligands to control cell proliferation, differentiation, and apoptosis. If any of its ligands bind to PTCH1, a receptor-coupled G protein, called Smoothened (SMO), is released, and the aforementioned gene is inhibited. SMO allows the release of Gli proteins from its Fusion Suppression Suppressor (SUFU), which leads to cell proliferation. The PTCH2 gene is also a tumor suppressor involved in this signaling pathway, but its association with the syndrome is rarely reported since this subtype has lower penetrance when compared to the genotype associated with PTCH1 [5,6]. This mutation can be inherited $(70-80 \%$ of cases) or result from new mutations (20 - 30\% of cases) [7]. The molecular genetic test can identify mutations in up to $75 \%$ of affected individuals and confirms the syndrome, although it is not essential for diagnosis. In most patients, clinical signs, imaging findings, histopathological features and family history will confirm the BCNS [6]. 
The most used method for diagnosis is the one proposed by Evans., et al. (1993) and Kimosis., et al. (1997), who listed major and minor criteria that should be met to confirm that a patient is affected by the syndrome [8]. At the moment, more than 100 minor criteria have been described in the literature. However, the presence of two major criteria or one major and two minor criteria is sufficient to establish the diagnosis [9].

Among the major criteria, we can mention first-degree family member with BCNS, presence of two or more basal cell carcinomas under the age of 20 years, presence of keratocystic odontogenic tumors (OKTs), presence of three or more palmoplantar depressions, changes in ribs (bifid, flattened or fused), calcification of the cerebral sickle, among others [10].

Among the minor criteria, the following stand out: macrocephaly, congenital malformations (cleft palate or lip, hypertelorism), prognathism, pontification of the Turkish saddle, vertebral anomalies, exotropia, mental retardation, cardiac fibroids, calcified ovarian fibromas, and many other conditions [10].

Given the wide variety of clinical manifestations of this syndrome, in this article we aim to recall its signs and symptoms, discuss its diagnostic process and illustrate it with two clinical cases, attended at the Federal University of Paraná (UFPR). It was also aimed at indicating its appropriate treatment, since the management of this condition requires the monitoring of a multidisciplinary team in the long term, due to its involvement of multiple systems, high rate of recurrence of lesions, and the late appearance of carcinomas.

\section{Literature Review}

For didactic purposes, the review of signs and symptoms was divided into 7 major areas: skin, skeletal, ophthalmic, cardiac, endocrine, neurological, and oral changes. Clinical signs tend to appear with advancing age and gradually increase, with the average time of diagnosis being between the second and third decade of life [3]. Some predisposing risk factors include excessive exposure to the sun, radiation, immunosuppression, and vitamin D deficiency [6].

\section{Skin changes}

The most common skin disorder among patients with this syndrome is basal cell carcinomas (BCCs), which occur in about $90 \%$ of patients. They vary in shape and color, usually appearing as a papule or plaque and measuring between 1 and 10 centimeters. Its major location is the face, the back, and the chest. However, they can appear in any area of the body and can appear more than 100 times in the same individual [11]. They often have a banal appearance and can be roughly mistaken for nevocytic nevi. In case of doubt, skin lesions should be biopsied [10].

The differential diagnosis is made with xeroderma pigmentosum, Bazex-Dupre-Christol Syndrome, Rombo Syndrome, and Muir Torre Syndrome. Besides, immunosuppression may be associated with the development of multiple BCCs and should be investigated $[1,12]$.

Due to the ineffectiveness of the mechanisms that repair DNA, ultraviolet radiation is a factor that requires care and patients should be instructed to adopt protective measures since excessive exposure increases the chance of developing BCCs. Caucasians tend to develop these lesions earlier than blacks, as the greater amount of melanin in the skin acts as a protective factor. Ionizing radiation should also be avoided, except when the patient has advanced lesions and radiotherapy is strictly necessary. When exposed to the aforementioned radiation, the aggressiveness of basal cell carcinomas can be increased [4].

Most articles usually condition the treatment of BCCs to the behavior and size of the lesions. When their number is limited and their staging is not advanced, surgical removal can be performed. In cases where there are multiple lesions, laser removal, topical chemotherapy and photodynamic therapy can be performed. In topical chemotherapy, the use of 5-fluorouracil and/or tretinoin is recommended and local reactions may occur. In photodynamic therapy, on the other hand, a prodrug is administered orally, intravenously or applied topically, and is subsequently activated by laser or UV radiation. Its main adverse effects are pain, scarring, and skin burns [9]. If left untreated, BCCs may eventually evolve with metastasis, reaching vital structures and leading the patient to death [1].

Another common alteration is depressions or palmoplantar depressions, which are small defects in the stratum corneum, caused by a partial or complete absence of this layer. They are observable with a tangential light and become dark if there is an accumulation of dirt. As age advances, they tend to become more evident. Immersing the region in water for 15 minutes can facilitate the visualization of this signal $[10,13,14]$. 
Skeletal changes

Among the skeletal changes, the temporoparietal pattern stands out, which results from an increase in cranial circumference $[9,13]$. Slight prognathism is commonly present [13]. Radiographic findings include changes in the ribs, which may be bifid, flattened, or fused, changes in vertebrae, such as hemivertebrae, fusion or elongation of the vertebral bodies, pectus excavatum, hand and foot defects (fourth short pasterns), Spengel deformity, pectoral deformity, and syndactyly [10].

The measurement of head circumference in adults is important for clinical evaluation. Its estimate is based on the patient's height and weight and follows the following formula: $0.063 *$ height + $0.040 *$ weight +42.618 . The sum of these values results in a percentage and values above the 90th percentile, macrocephaly should be considered [5].

Imaging exams will vary according to the situation and may include panoramic, skull and chest radiography. However, since these patients are highly sensitive to ionizing radiation, their use should be minimized [10].

\section{Ophthalmological changes}

The pathologies that commonly affect these patients are periocular basal cell carcinomas, strabismus, congenital cataracts, coloboma of the iris, glaucoma, nystagmus and congenital blindness. One of the most reported findings in the literature is hypertelorism, a congenital malformation that culminates in increasing the distance between the eyes and constitutes one of the minor criteria for the diagnosis of the syndrome when in grade II or III [10].

To assess the eyes and orbits, one should start by measuring the intercantal and interpupillary distances. The average value of this difference is usually $4 \mathrm{~mm}$, and black people often have values above the average. The classification of ocular hypertelorism is made after measurement on a frontal teleradiography, being classified as grade I (30 - $34 \mathrm{~mm}$ ), grade II (34 - $39 \mathrm{~mm}$ ), or grade III (40 mm or more) [5].

\section{Cardiac changes}

Cardiac fibroids, which affect 3 to $5 \%$ of patients with BCNS, stand out. They should be investigated when it is possible to observe irregularities in the cardiac contour, cardiomegaly, calcifications in the myocardium or unexplained heart failure. The most common complications are severe arrhythmias, disturbances in driving and congestive heart failure. It is indicated that, as soon as the syndrome is detected, a transthoracic echo-Doppler cardiogram is performed to detect whether this alteration is present [5].

\section{Endocrine changes}

Hypogonadism often occurs in men and calcifications in the ovaries of women. Ovarian tumors are usually benign, asymptomatic and do not interfere with fertility. They rarely undergo malignant transformation. When there is a need for craniospinal irradiation, the ovaries that contain the tumors must be monitored [15]. It is also possible to find in the literature articles that report patients with Gorlin-Goltz Syndrome and other comorbidities of an endocrinological order, such as Diabetes Mellitus 2 and arterial hypertension systemic [5].

\section{Neurological changes}

Among the neurological changes, the following may occur: mental retardation, calcifications of the dura mater, calcifications of the cerebral sickle, agenesis of the corpus callosum, congenital hydrocephalus and medulloblastoma [15].

Medulloblastoma is a malignant brain tumor of the posterior fossa, which appears between 1 and $10 \%$ of patients with BCNS. In the general population, they appear between 7 and 26 years old. However, in this syndrome, It usually presents in the first two years of life and can be diagnosed in children with an average age of 5 years [15]. It preferentially affects males, with a prevalence of 3: 1 among men and women [12]. When associated with this syndrome, they have a dermoplasic phenotype, which some studies suggest is related to a better prognosis [4].

Medulloblastoma is a potential cause for early deaths in these patients and, therefore, it is important to identify this pathology early through imaging tests, as is the case with cerebral magnetic resonance [10].

\section{Oral changes}

The odontogenic keratocyst is part of the triad that characterizes the syndrome and was reclassified as a keratocystic odontogenic tumor (OKT) in the last book of the World Health Organization (2017), due to its aggressive behavior, its histology, and its genetics [1,2]. OKT is present in $65-75 \%$ of cases and is often the first detectable manifestation of the syndrome. It can be present in a number from 1 to 30 , with an average of $5[12,16]$. It is more common in the jaw, appearing in up to $70 \%$ of cases, with the majority 
occurring in the posterior region of the body and the branch [1]. In the upper arch, it is noticed mainly in the third molar region, followed by the canine region $[3,10]$. Commonly, OKT in patients with this syndrome is multiple and the lesions are asymptomatic. When they reach a large size, patients often report swelling, mild pain and teeth adjacent to the lesion can be dislocated $[1,3]$.

Radiographically, they appear as uni or multilocular image, commonly associated with a withheld tooth, although this is not a rule [8]. Aspiration puncture is an important tool if this lesion is suspected and reveals a thick, straw-colored liquid and with a large amount of keratin, which gives it an aspect described by some authors as "melted candle" [1]. From a microscopic point of view, OKTs usually have satellite cysts and large areas of mitosis. Solid islands of epithelial proliferation are also frequently found. The parakeratinized variant is the most prevalent in the cases of Gorlin-Goltz Syndrome [8].

The differential diagnosis of OKT should include the dentigerous cyst, the lateral periodontal cyst, the unicystic ameloblastoma, odontogenic fibromyxoma, and residual cysts [1].

The treatment of OCD vary, depending on the proportion of the lesion, its location, extent, age of the patient, and whether it is primary or recurrent. In small tumors, the treatment of choice is simple enucleation associated with curettage, which aims to remove 1 to $2 \mathrm{~mm}$ of tissue. Other options are enucleation with peripheral osteotomy or a bloc resection, the latter approach is considered the most aggressive. For large injuries, conservative options include Carnoy's solution therapy, marsupialization of the cysts with cryotherapy, followed by secondary enucleation or decompression. General anesthesia is a management option when the number of lesions is high or there is a risk of mandibular fracture during the procedure [1]. The recurrence rate of isolated keratocystic odontogenic tumors is approximately $30 \%$, while in patients with SGG the recurrence rate is $82 \%$ [9]. This high recurrence rate can be explained by the incomplete removal of the cyst wall or satellite cysts not removed $[3,12]$.

Follow-up should be semiannual in the first five years, where the recurrence rate is higher and yearly after that period $[15,16]$. The request for an annual panoramic radiograph must be included in the treatment protocol [1].

Other oral tumors already described in patients with this syndrome include ameloblastoma, myxoma, fibrosarcoma, squamous cell carcinoma, adenoid cystic carcinoma, and lymphoma, 9 while other reported developmental defects are cleft lip/palate, ectopic teeth, impacted teeth, agenesis dental, malocclusion, mandibular prognathism, atresia palate, skeletal open bite and hyperplasia of the coronoid process $[12,16]$.

\section{Case Report 1}

A black Brazilian 9-year-old man has been followed at the UFPR outpatient clinic, since the $15^{\text {th }}$ day of life, due to the presence of a unilateral right transforaminal fissure, according to Spina's classification (1973).

During routine periapical radiography for the treatment of a carious lesion, the presence of a radiolucent lesion in the region of tooth 84 was discovered. The patient was referred to the UFPR clinic for evaluation of the lesion, where panoramic radiography was requested, in which we found the presence of a radiolucent lesion involving the region of the body and right branch, as well as the mandibular symphysis (Figure 1).

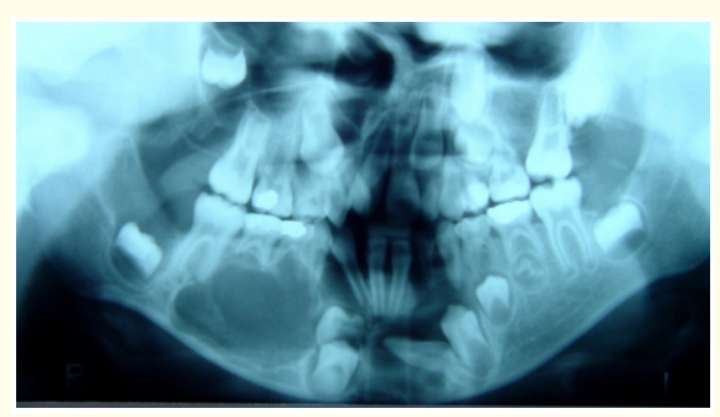

Figure 1: Panoramic radiograph showing radiolucent lesion, with a unilocular aspect that extends from the region of the body and mandibular branch to the symphysis on the right side.

The mandibular lesion was removed by enucleation (Figure 2 ), under general anesthesia, and the patient was referred for histopathological examination at the pathology department of the Federal University of Pelotas, with the diagnosis of a keratocystic odontogenic tumor.

Because of the lesion finding and the patient's clinical characteristics observed in the clinical examination (prominent frontal and parietal hump, hypertelorism, wide nasal bridge, and difficulties in school follow-up) (Figure 3), it was suspected that he had Gorlin-Goltz Syndrome. 


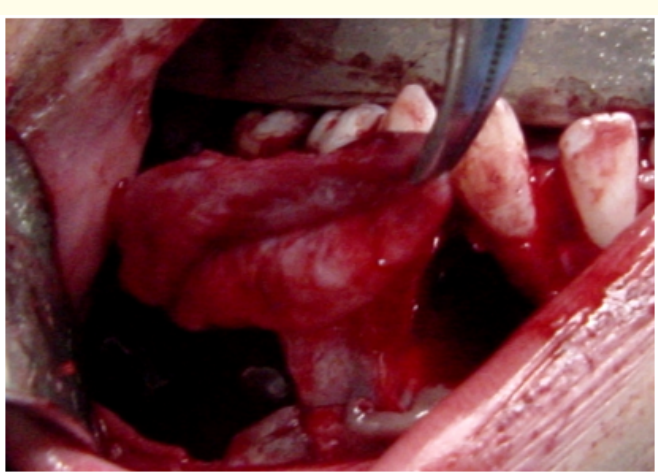

Figure 2: Removal of the lesion in the mandible during surgery. It is possible to observe the presence of a capsule surrounding the lesion.

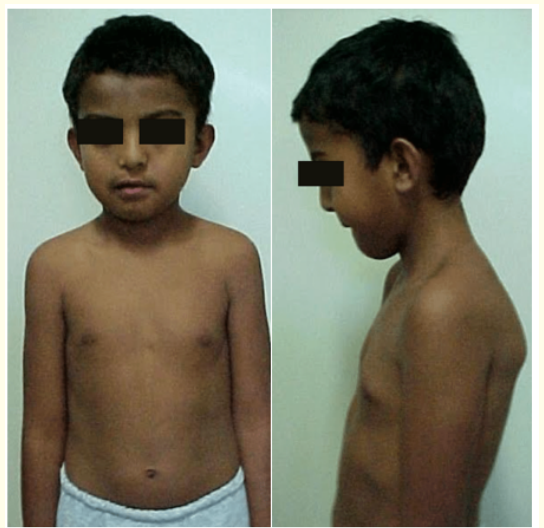

Figure 3: Frontal and lateral view of the patient, where it is possible to observe prominent frontal and parietal bosses, hypertelorism, and a wide nasal bridge.

Then chest, skull, and hand radiographs were requested. The bone structures of the hand and the skull did not present abnormalities; however, in the thoracic region, anatomical variations were observed in the $4^{\text {th }}$ and $5^{\text {th }}$ costal arch on the right and the $3^{\text {rd }}$ arch on the left, in addition to a slight increase in the cardiac area.

After confirming the diagnosis, the patient underwent annual follow-up by the oral maxillofacial surgery department I. Two years after the first intervention, a recurrence of the lesion was located in the right region of the mandible, involving teeth 47 and 48 , as well as a new one. injury involving teeth 43 and 44, still forming. On the left side of the mandible, new lesions involving teeth 73 and 74 and germs from teeth 34 and 35 were found. All lesions were removed by curettage and cauterization with an electric scalpel at the edges of the wounds, together with the removal of elements $73,74,34$, 35, 43, and 44 (Figure 4). The lesions were separated according to the location where they were found and sent for histopathological examination in the pathology sector of PUC-PR, with the diagnosis of parakeratinized keratocystic odontogenic tumor.

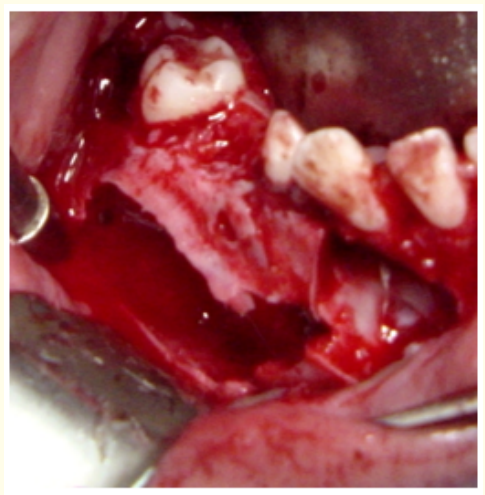

Figure 4: The appearance of the cavity after removal of the recurrent lesion.

After researching the family history, the patient's mother was unable to inform whether there are pre-existing cases in the family.

\section{Case Report 2}

Caucasian Brazilian 19-years-old patient was referred to the Oral and Maxillofacial Surgery outpatient clinic of the Federal University of Paraná for investigation of intraosseous lesions observed on panoramic radiography. The patient did not report symptoms and did not present asymmetry of the face. In the intraoral examination, an intact and normal colored mucosa was observed, with loss of the vestibule fundus at the height of the elements 36 and 37 . In the maxilla region, the vestibule fundus was erased superior to the right premolars and molars. Radiographically, it was possible to observe an osteolytic lesion in the maxilla (Figure 5). A computed tomography scan was then requested for better visualization of the lesions.

On tomography, it was observed that the lesion in the maxilla was extensive, with loss of the lateral wall of the maxillary sinus and involvement of the teeth $13,14,15,16$ and 17 . In the mandible, the lesion presented an expansion of the bone cortices, without rupture and involving multiple elements (Figure 6 and 7). 


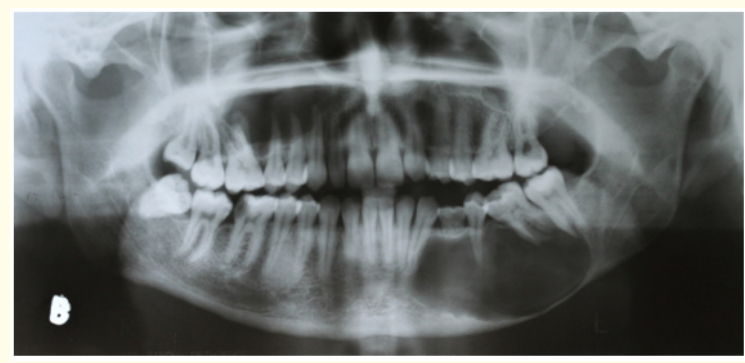

Figure 5: Panoramic radiograph showing two radiolucent lesions with a unilocular aspect, located in the right maxilla, in the region of the premolars and molars, and the left mandible, extending from the distal of the canine to the lower second molar.

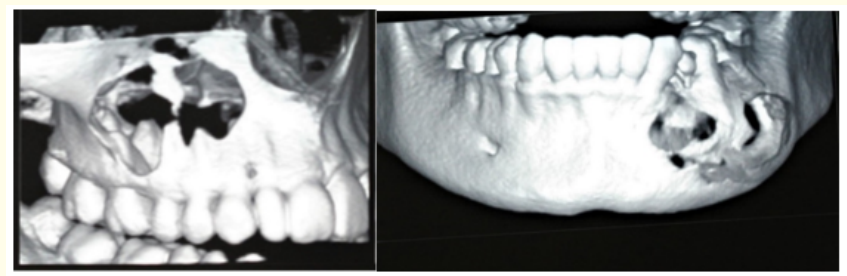

Figure 6 and 7: Three-dimensional reconstruction of the case using computed tomography. It is possible to observe the extension of the lesion in the maxilla, with consequent loss of the lateral wall of the maxillary sinus. In the reconstruction of the mandible, there is an expansion of the cortical bone.

After planning, it was decided to submit the patient to general anesthesia, with aspiration puncture followed by excisional biopsy of the identified lesions.

In the puncture of the maxilla lesion, bloody material was collected (Figure 8). In the jaw injury, there was a lot of keratin (Figure 9). Both lesions had a capsule and were well delimited. In the jaw injury, it was also possible to observe the intact lower alveolar nerve, however, adhered to the capsule (Figure 10). After complete removal (Figure 11 and 12), all material collected was sent for anatomopathological examination, which confirmed the diagnostic hypothesis of a keratocystic parakeratinized odontogenic tumor. Given this result, an investigation was started on the possible syndromic condition of the patient.

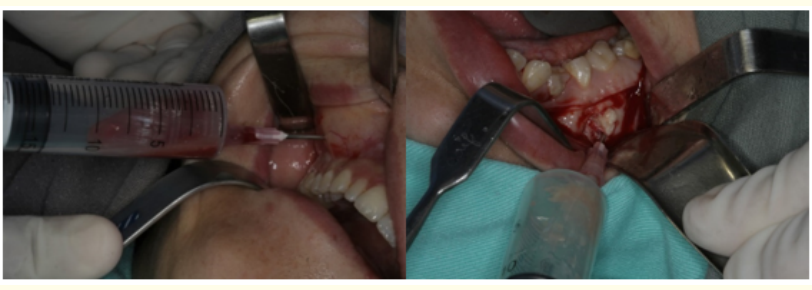

Figure 8 and 9: Aspiration puncture of the lesions. In the maxillary puncture, bloody material was collected, while in the mandibular lesion, the material was more keratinized.

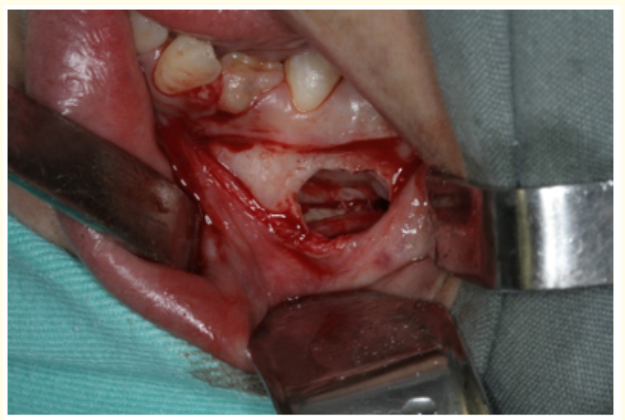

Figure 10: The inferior alveolar nerve is seen in its entirety, during the removal of the lesion.

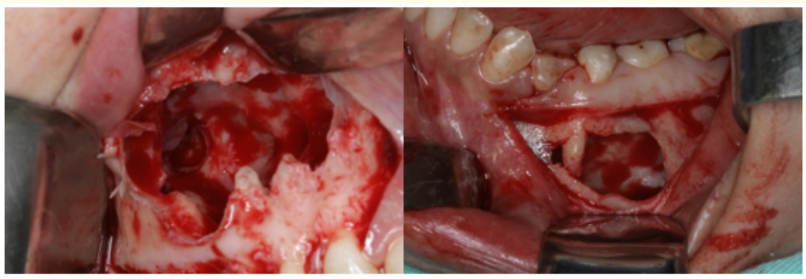

Figure 11 and 12: The appearance of the cavities in the maxilla and mandible, respectively, after the complete removal of the lesions.

Chest radiography, cardiological and dermatological evaluation were then requested. On chest radiography, it was possible to observe the presence of bifid ribs (Figure 13). Although no abnormalities were noticed in the cardiological evaluation, the dermatology service detected nevi in the region of the face (Figure 14) and the 
back. The biopsy was performed and confirmed the development of basal cell carcinoma. Because of the findings, the hypothesis that the patient was a carrier of Gorlin-Goltz Syndrome was confirmed.

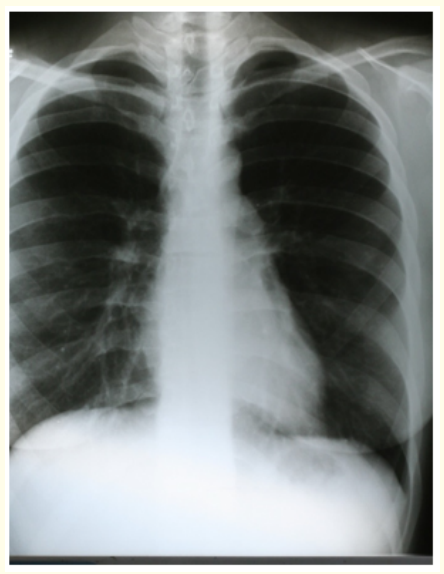

Figure 13: Chest $x$-ray, where it is possible to observe the presence of bifid ribs.

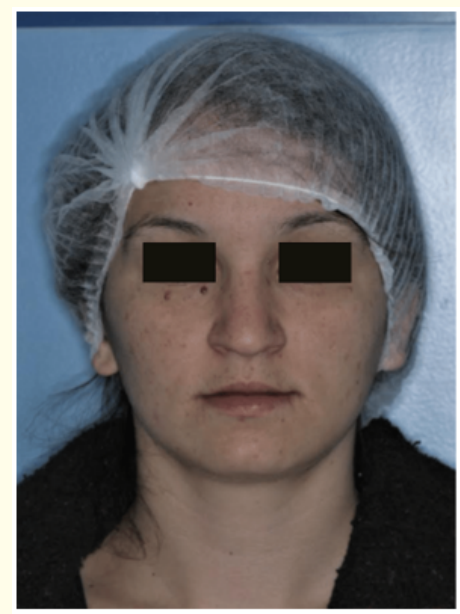

Figure 14: Frontal view of the patient, showing multiple nevi in the face region.

At the moment, she is being monitored.

\section{Discussion}

Gorlin-Goltz syndrome is a pathological clinical entity, which is mainly characterized by the presence of multiple basal cell carci- nomas, keratocystic odontogenic tumors and skeletal anomalies. It is an autosomal dominant condition, with high penetrance and variable expressiveness.

This variable expressiveness culminates in different clinical and radiographic presentations in the affected patients, which complicates their diagnosis, which, many times, ends up being established late [10]. The genetic test is unable to detect changes in all individuals and the absence of family history corroborates this difficulty $[6,13]$.

Chorionic villus or amniotic fluid sampling to assess the genetic mutation can be performed during pregnancy. Tests such as ultrasound can be used to detect cleft palate, macrocephaly and other tumors associated with the syndrome [6].

In families with the diagnosis suggested by family history, newborns should be examined for mandibular prognathism, cleft lip or palate, abnormalities in the ribs, macrocephaly, syndactyly, cataracts and other characteristic manifestations [6].

In the first clinical case presented, no basal cell carcinomas were found, probably due to the patient's young age and skin pigmentation, which gives him greater protection. The palmar and plantar depressions, common to patients with this syndrome, were also not observed in the case in question. However, the patient had two major criteria (the presence of keratocystic odontogenic tumors and changes in the ribs) and several minor criteria (frontal hump, cleft lip/palate, hypertelorism), which are sufficient to close the diagnosis of the syndrome, according to the one established by Evans., et al. (1993) and Kimosis., et al. (1997).

In the second clinical case, the presence of multiple keratocystic odontogenic tumors was the main element that led to the diagnostic hypothesis of Gorlin-Goltz Syndrome. Later, the finding that the patient had bifid ribs and that she had developed a basal cell carcinoma under the age of 20 years closed the classic triad that characterizes the syndrome, meeting three major criteria.

The presence of basal cell carcinomas and multiple keratocystic odontogenic tumors are practically a pathognomonic sign of BCNS [11]. It is unanimous among authors that the presence of a single OKT in an individual under the age of 20 should be an indication of investigation for Gorlin-Goltz Syndrome [4]. Therefore, the relevance of the maxillofacial surgeon in the identification and treat- 
ment of this pathology is highlighted, since, in many cases, the presence of this lesion will be the first detectable sign of the syndrome [17].

The OKT findings are very characteristic in this context and were compatible for the first and second patients, due to the asymptomatic presence of multiple lesions in young individuals, who obeyed the typical imaging and histological characteristics. Treatment can vary according to the proportions of the lesion and the patient's characteristics, from surgical approaches for small lesions, such as simple enucleation associated with curettage, to more conservative techniques for large lesions, such as marsupialization, followed by decompression or enucleation. secondary. The recurrence of injuries, which is usually higher in patients with BCNS, especially in the first 5 years after removal, was also significant in the first case presented. Given the above, the monitoring must be semiannual in the first five years and annually after that period. The annual request for a panoramic radiograph must be included in the monitoring protocol $[3,17]$.

In general, the clinical findings of the first case partially contradict the literature, as he is a melanoderma patient and his first clinical manifestations are multiple OCDs, which became evident after the first decade of life and enabled early diagnosis, which allows also to identify early important changes that can be lifethreatening for patients, such as medulloblastoma and cardiac abnormalities. This indicates that even patients from an ethnic group in which the syndrome is diagnosed less frequently should be carefully investigated. In the second case presented, the patient also had as the first detectable manifestation of the syndrome multiple keratocystic odontogenic tumors, which emphasizes the relevance of the maxillofacial surgeon in the diagnosis and treatment of BCNS. The request for a dermatological examination was crucial for the detection of basal cell carcinoma, showing that multidisciplinarity is fundamental in the management of the syndrome and, in turn, directly impacts the patient's quality of life.

The prevention of basal cell carcinomas involves changing habits, with protection from UV and ionizing radiation. Vitamin D supplementation can be recommended. The use of photodynamic therapy and chemoprevention is not yet well established [6]. Currently, efforts are being made to develop new molecular therapies for the treatment of skin lesions, especially for application in cases where the surgical approach is impractical [7].
The condition of patients requires attention and long-term follow-up by a multidisciplinary team is essential. It must include the maxillofacial surgeon, dermatologist, oncologist, neurologist, cardiologist, among other professionals. In many cases, psychological counseling is important to assess the emotional impact of the syndrome on the patient [6]. The geneticist must also be present, since genetic counseling is necessary, suggesting to patients who avoid having children since there is a high risk of offspring involvement [9].

\section{Conclusion}

Gorlin-Goltz Syndrome demands multidisciplinary attention, due to its wide range of clinical manifestations. Once the diagnosis is established, treatment consists of managing its consequences. The monitoring of patients with this entity must be constant, due to the appearance of new manifestations throughout life. The early detection of lesions such as medulloblastoma and basal cell carcinomas can be definitive in the quality of life of patients. In this context, the maxillofacial surgeon plays a fundamental role in the identification and treatment of BCNS, since, often, keratocystic odontogenic tumors can become the first detectable manifestation of the syndrome.

\section{Bibliography}

1. Kuhn-Dall'magro A., et al. "Síndrome de gorlin-goltz - relato de casos". RFO UPF 19.2 (2014): 239-244.

2. Gorlin RJ and Goltz RW. "Multiple nevoid basal-cell epithelioma, jaw cysts and bifid rib. A syndrome". The New England Journal of Medicine 262 (1960): 908-912.

3. Stolz ASB., et al. "Proservação pós-operatória de paciente com Síndrome de Gorlin-Goltz - relato de caso". Revista Sul-Bras Odontology 7.2 (2010): 226-230.

4. Val FS., et al. "A importância do cirurgião-dentista na detecção da Síndrome do Carcinoma Nevoide Basocelular: relato de caso". Revista Brasileira de Odontologia, Rio de Janeiro 67.1 (2010): 34-38.

5. Drumond JPN., et al. "The Gorlin-Goltz Syndrome: diagnosis of a case associated with heart disease and type 2 diabetes mellitus". Revista Brasileira de Cirurgia Plástica 31.4 (2016): 578-582.

6. John Am, Schwartz Ra. "Basal cell naevus syndrome: an update on genetics and treatment". British Journal of Dermatology 174.1 (2016): 68-76.

Citation: Juliane Maria Iagnes Prestes and João Luiz Carlini. "Gorlin-Goltz Syndrome: Literature Review and Report of Two Clinical Cases". Acta Scientific Dental Sciences 5.5 (2021): 86-94. 
7. Abreu JM., et al. "Síndrome de Gorlin-Goltz: diagnóstico e hipóteses de tratamento". Revista Científica da Ordem dos Médicos 30.5 (2017): 418-421.

8. Silva QCL., et al. "Diagnóstico precoce e tratamento da síndrome de Gorlin-Goltz: acompanhamento de oito anos". Revista Cubana de Estomatología 57.1 (2020).

9. Oliveira Sp., et al. "Síndrome de Gorlin-Goltz: um relato de caso". International Journal of Science Dentistry, Rio de Janeiro 45 (2016): 1-10.

10. Medeiros L., et al. "Revisão Bibliográfica a Propósito de um Caso Clínico". Revista Portuguesa de Estomatologia, Medicina 47 (2006): 25-32.

11. Fini G., et al. "Nevoid basal cell carcinoma syndrome (GorlinGoltz syndrome)". Case Report G Chir 34.5-6 (2013): 176-179.

12. Safronova Mm., et al. "Síndrome de Gorlin-Goltz - revisão das características neurorradiológicas e maxilofaciais ilustradas com dois casos". Acta Médica Portuguesa 23 (2010): 11191126.

13. Pierro Vss., et al. "Clinical and oral findings in an Afro-Brazilian family with Gorlin-Goltz syndrome: case series and literature review". Special Care in Dentistry 35.1 (2014): 43-50.

14. Shanley S., et al. "Nevoid basal cell carcinoma syndrome: review of 118 affected individuals". American Journal of Medical Genetics 50 (1994): 282-290.

15. Evans Der., et al. "Complications of naevoid basal cell carcinoma syndrome: results of a population based study". Journal of Medical Genetics 30 (1993): 460-464.

16. Khaliq MIU., et al. "Keratocystic odontogenic tumors related to Gorlin-Goltz syndrome: A clinicopathological study". Journal of Oral Biology and Craniofacial Research 6.2 (2016): 93-100.

17. Ahuja S., et al. "Multiple odontogenic keratocyst: A case report and review of literature". Journal of Dental Specialities 8.1 (2020): 21-25.

\section{Assets from publication with us}

- Prompt Acknowledgement after receiving the article

- Thorough Double blinded peer review

- Rapid Publication

- Issue of Publication Certificate

- High visibility of your Published work

Website: www.actascientific.com/

Submit Article: www.actascientific.com/submission.php

Email us: editor@actascientific.com

Contact us: +919182824667 